\title{
Die tägliche Speisung des Assur (ginā’u) und deren politische Bedeutung
}

\author{
Stefan M. Maul
}

HEIDELBERG

Bei den Ausgrabungen der Deutschen-Orientgesellschaft in Assur untersuchten Walter Andrae und sein Team im Sommer 1911 die noch anstehenden Reste der Gebäude, die einst den großen Vorhof des Assur-Tempels umrahmt hatten. ${ }^{1} \mathrm{Da}-$ bei stießen die Archäologen in dem nordwestlichsten Raum des Nebengebäudes, das sich im Südwesten an den Tempel anschloß (Abb. 1, Raum 3'), auf den bedeutendsten und weitaus umfangreichsten Fund von Archivalien aus dem Bereich des Haupttempels des assyrischen Reiches. ${ }^{2}$

Als dieser in mittelassyrischer Zeit als eine Art Rampe genutzte, von Nordwest nach Südost stark abschüssige Raum umgebaut wurde, hatte man sich die Mühe gespart, den Raum ganz auszuleeren, bevor man damit begann, den tief liegenden südlichen Bereich durch Aufschüttungen zu erhöhen. So blieben dort, unweit der südöstlichen Schmalwand, insgesamt zehn tönerne Töpfe liegen, in denen Keilschrifturkunden abgelegt waren (Abb. 2), die man offenbar für so unwichtig erachtete, daß man darauf verzichtete, sie sicherzustellen.

Die eigens zur Aufbewahrung der Dokumente hergestellten und zum Teil sogar beschrifteten Gefäße ${ }^{3}$ (Abb. 3) enthielten insgesamt mehr als 650 Dokumente. ${ }^{4}$ Man war auf jene Aufzeichnungen der Opferverwaltung des Assur-Tempels gestoßen, die O. Pedersén in seinem Buch Archives and Libraries in the City of Assur als das mittelassyrische Archiv M 4 beschrieb. ${ }^{5}$ H. Freydank machte in den vergangenen

1. Zu den Ausgrabungen im Gebiet des Assur-Tempels siehe A. Haller und W. Andrae, Die Heiligtümer des Gottes Assur und der Sin-Šamaš-Tempel in Assur, WVDOG 67 (Berlin: Mann, 1995). Die mittelassyrischen Bauphasen sind dort auf den Seiten $37 \mathrm{ff}$. behandelt.

2. Siehe O. Pedersén, Archives and Libraries in the City of Assur [im folgenden: ALA], Part I (Uppsala: Almqvist \& Wiksell, 1985), 43-53.

3. Von den zehn Gefäßen waren drei mit Beschriftungen versehen: 1.) VA Ass. 1138 (Fundnummer: Ass. 18763): siehe Abb. 3 sowie O. Pedersén, Katalog der beschrifteten Objekte aus Assur (Saarbrücken: SDV 1997), 126 mit weiterführender Literatur. 2.) VA 5035 (Fundnummer: Ass. 18766) mit dem Verweis, daß es Dokumente enthalte, die sich "auf den kakardinnu und den Ölpresser des Assur-Tempels" beziehen unter der Aufsicht des "Ezbu-lēšer, des Opferaufsehers ( $r a b$ ginā'e $)^{\text {": }}$ siehe O. Pedersén, Kata$\log$ der beschrifteten Objekte aus Assur, 126 mit weiterführender Literatur. 3.) VA 5046 (Fundnummer: Ass. 18827), mit dem Verweis, daß es "gesiegelte Urkunden der [Brauer] des Assur-Tempels" enthalte, die unter der "Aufsicht des "Ezbu-lěšer, des Opferaufsehers ( $r a b$ ginä’e)" standen: siehe O. Pedersén, Katalog der beschrifteten Objekte aus Assur, 126 mit weiterführender Literatur.

4. Siehe O. Pedersén, ALA I, 43.

5. O. Pedersén, $A L A$ I, 43-53. Der schon durch die Fundsituation hervorgerufene Eindruck, daß die in den zehn Gefäßen aufbewahrten Dokumente kein vollständiges, in sich geschlossenes Archiv 


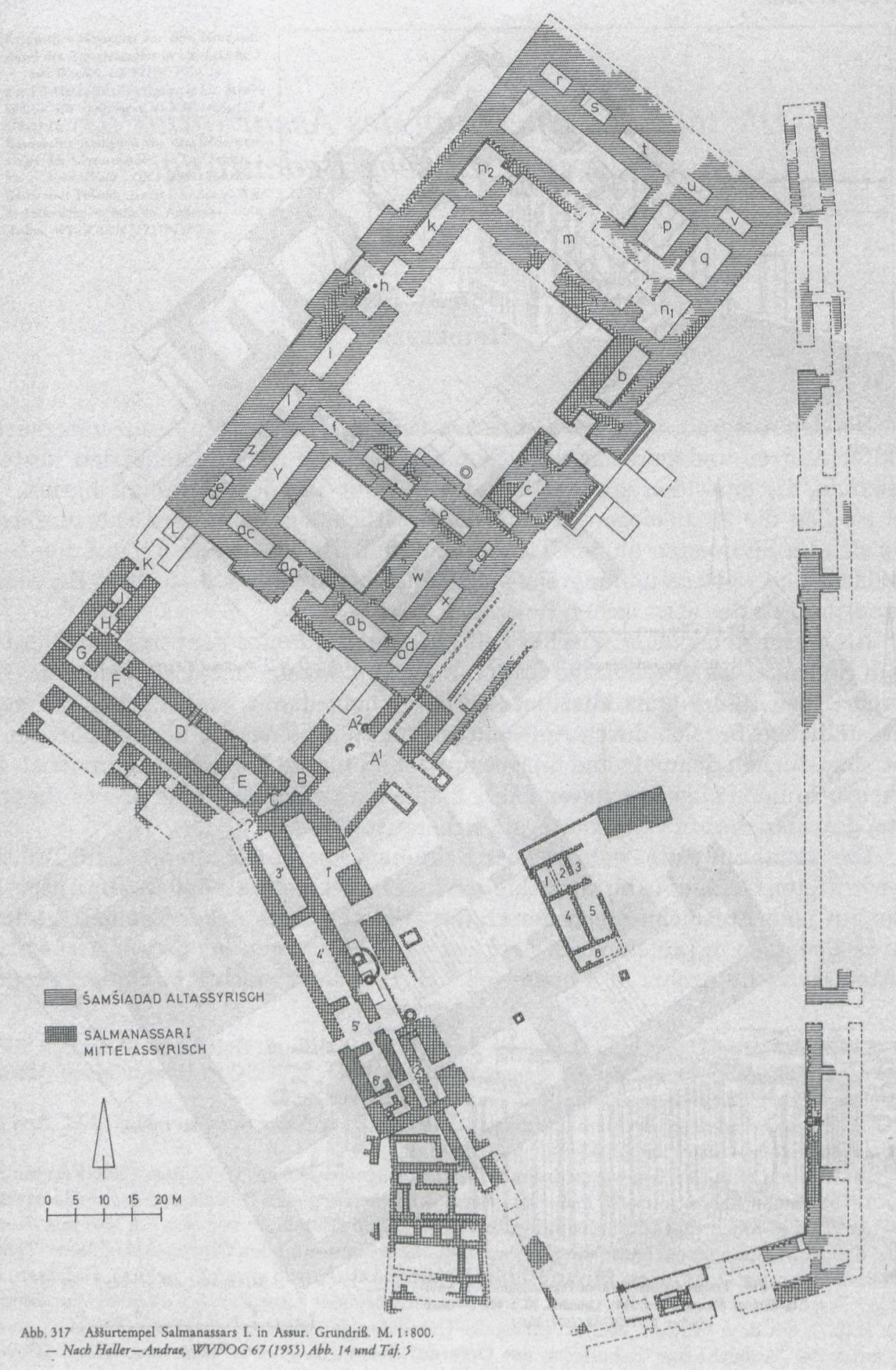

Abb. 1. Der Assur-Tempel in mittelassyrischer Zeit (nach E. Heinrich, Die Tempel und Heiligtümer im alten Mesopotamien, Abb. 317). 


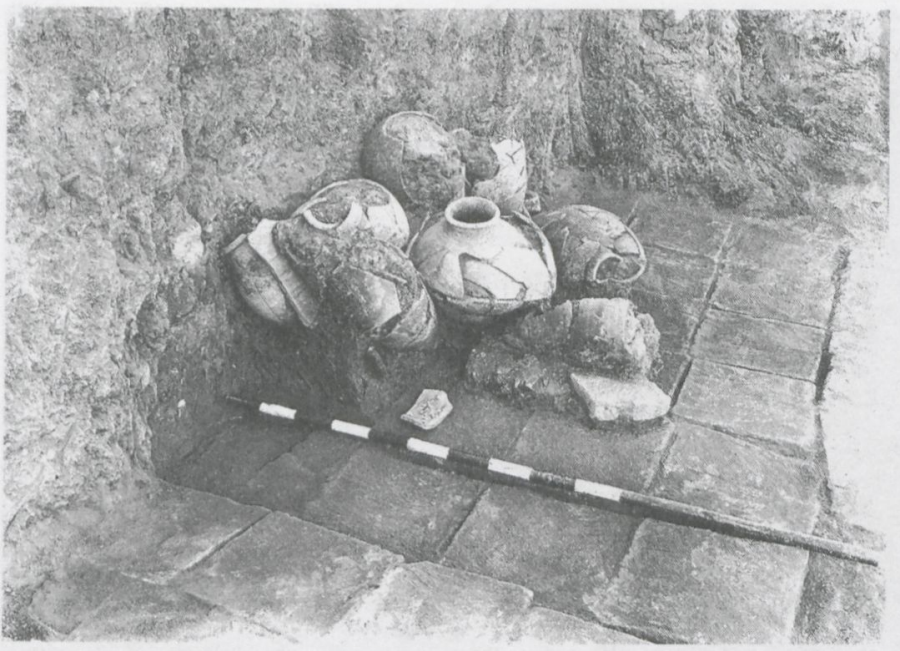

Abb. 2. Die mittelassyrischen Tontafeldepots aus der Opferverwaltung des AssurTempels ("M 4") in Fundlage (Assur-Photo 5685).

Jahren einen beachtlichen Teil dieser Dokumente-zumindest in keilschriftlichen Autographien-in den Bänden der Reihe Mittelassyrische Rechtsurkunden und Verwaltungstexte (im folgenden: MARV) zugänglich. ${ }^{6}$

Der Archivbestand dokumentiert im wesentlichen die Aktivitäten im Umfeld von vier Verwaltungsbeamten, die im Verlauf des 12. und frühen 11. vorchristlichen Jahrhunderts in ihrem Amt aufeinander folgten und den Titel "Aufseher des regelmäßigen Opfers« (ša muhhi gină’e bzw. rab ginā’e) trugen. Aba-lā-1̄de, Sînuballiț und Sîn-nādin-apli dürften nacheinander als ša muhhi ginā’e "vom Ende der Tukultī-Ninurta-Zeit wenigstens bis in die Regierungszeit Aššur-dāns I." 7 tätig gewesen sein. Ihr Nachfolger Ezbu-lēšer versah sein Amt ( $r a b$ ginā'e) unter Tiglatpileser I. (1114-1076 v. Chr.). Der weitaus größte Teil des erhaltenen Archivbestandes stammt aus der Regierungszeit dieses Königs.

darstellen, sondern nichts weiter sind als die zufallsbedingten Überbleibsel einer ursprünglich systematischer zusammengestellten Sammlung von Schriftstücken, bestätigt sich bei der Auswertung der Dokumente.

6. H. Freydank, MARV 1 (= VS 19; Berlin: Akademie-Verlag, 1976), Texte Nr. 11, 16, 21, 25, 33, 49, 52, 56, 62, 66, 70 und 73; MARV 2 (=VS 21; Berlin: Akademie-Verlag, 1982), Texte Nr. 2, 3, 8, 13, 14, 18, 21 und 24; MARV 3 (= WVDOG 92; Berlin: Mann, 1994), Texte Nr. 6, 9, 14, 21, 22, 24-32, 34-42, 44, 45, 47-52, 55, 60, 61, 76, 84-86; MARV 5 (= WVDOG 106; Berlin: Mann, 2004), Texte Nr. 1, 2, 6-14, 16-29, 31-44, 48-51, 54-55, 57, 58, 60, 62-68, 70, 73 und 76; MARV 6 (= WVDOG 109; Saarwellingen: SDV, 2005), Texte Nr. 1-90; MARV 7 (= WVDOG 111; Saarwellingen: SDV, 2006), Texte Nr. 1-102; MARV 8 (= WVDOG 119; Wiesbaden: Harrassowitz, 2007), Texte Nr. 2, 6, 8, 9, 20, 22, 24, 25-27, 29, 30, 32, 35, $36,40,46,48,49,50,56,60,61,62,64,66,68,69,74,75,78,79,85,88,91,92,94,95$ und $96 ; M A R V 9$ (= WVDOG 125; Wiesbaden: Harrassowitz, 2010), Texte Nr. 1, 2, 6, 8-11, 14-17, 19, 21-25, 27, 30-32, $34,41,42,46,49,50,63,69,75,76,80,81,86,90,95-98,100,103-108,110$ und 112-116. Editionen von einem kleineren Teil dieses Archivs hat H. Freydank in seinem Aufsatz: "Das Archiv Assur 18764", AoF 19 (1992), 276-321 vorgelegt. In diesem Aufsatz wurden die Tafeln ediert, die in dem in Abb. 3 gezeigten Topf gefundenen wurden. Hingegen folgt die Veröffentlichung der Tafeln aus dem Archiv der Opferverwalter in der Reihe MARV keinem ersichtlichen Ordnungsprinzip. Dem von H. Freydank mit AoF 19, 276-321 gegebenen Beispiel folgend, sollte man bei zukünftigen Untersuchungen darauf achten, daß stets jene Tafeln als zusammengehörig betrachtet und behandelt werden, die gemeinsam in einem Tontafelgefäß aufbewahrt wurden. Den Schlüssel zu derartigen Untersuchungen liefert O. Pedersén mit seiner nach Tontafelbehältern geordneten Zusammenstellung der Archivalien in ALA I, 48-52.

7. H. Freydank, $A o F 19$ (1992), 278. Zu den vier Beamten Aba-lā-īde, Sîn-uballiṭ und Sîn-nādinapli und Ezbu-lēšer siehe ebd., 276-278 mit Anm. 10. 


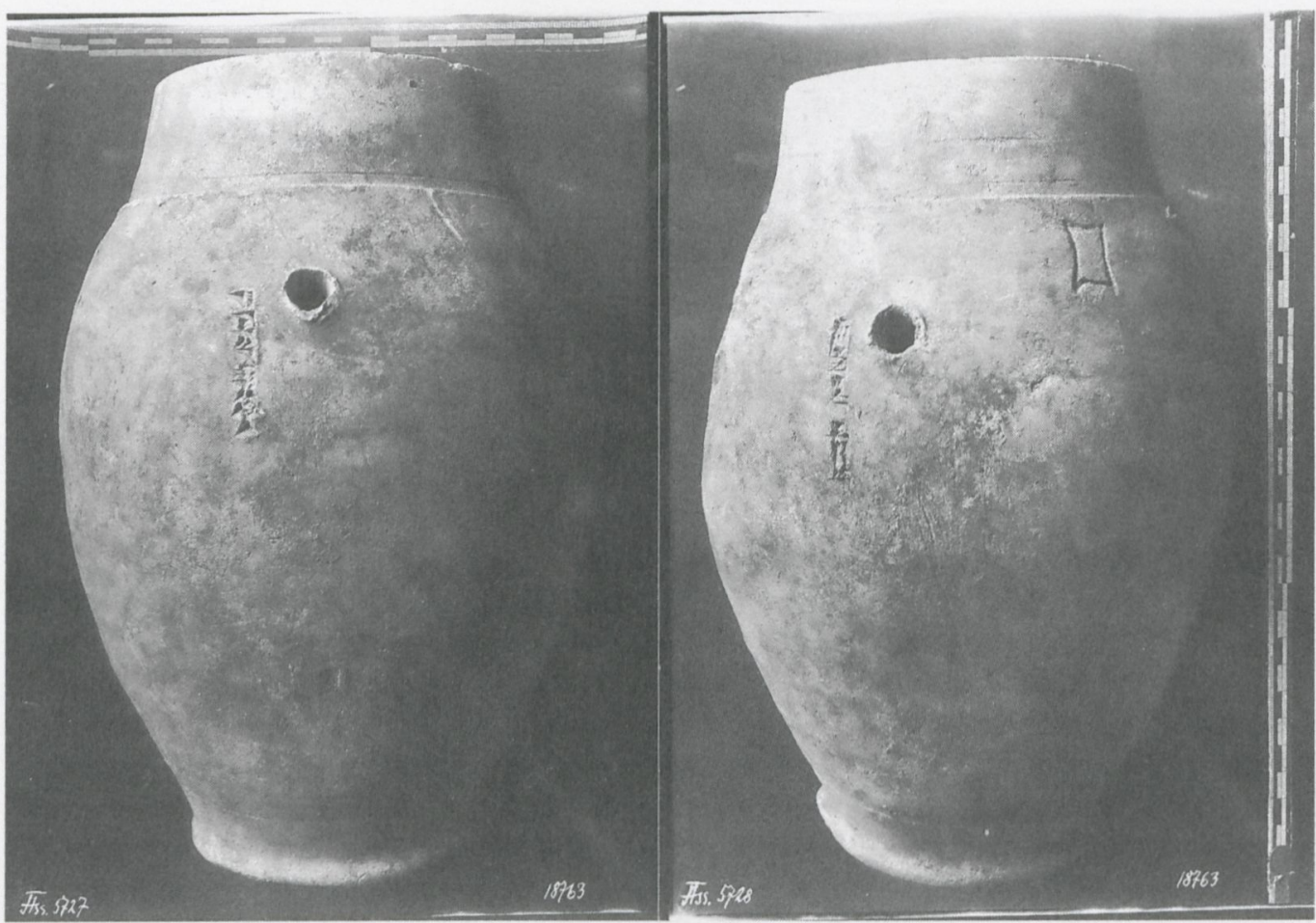

Abb. 3. Mit dem Namen des Eigners (šá l.d Šamaš-aha-ēriš / mār Ri-iš-d Marduk) beschrifteter und mit Lüftungslöchern versehener Topf für die Aufnahme eines Tontafelarchivs. Das Piktogramm einer Tontafel (?) sollte offenbar die der Keilschrift Unkundigen auf den Inhalt des wohl ursprünglich verschlossenen Topfes verweisen.

Die in dem Nebengebäude des Assur-Tempels entdeckten Urkunden dokumentieren bis in Einzelheiten Eingang, Verwertung und Verwendung einiger Güter, die für die von den Assyrern gina $\bar{a}^{\prime} u$, »Regelmaß«, genannte tägliche Speisung der Götter des Assur-Tempels verwendet wurden. Während Getreide, Sesam, Früchte und Honig hierbei eine hervorragende Rolle spielten, ist eigentümlicherweise in keinem der aus der Opferverwaltung des Assur-Tempels stammenden Dokumente von Opfertieren oder von Fleischlieferungen die Rede, obgleich doch auch in mittelassyrischer Zeit das blutige Opfer von großer Wichtigkeit war. ${ }^{8}$

Die Urkunden belegen, daß die im Assur-Tempel verbuchten Naturalienlieferungen als "Abgabe $(m a(d) d a t t u)$ « betrachtet wurden ${ }^{9}$, welche alle Provinzen des Reiches regelmäßig zu erbringen hatten. Der Abgabeverpflichtung kam dabei eine

8. Dies zeigt sich allein schon in der tabellenförmigen Aufstellung aus mittelassyrischer Zeit über Häute von weit mehr als 5000 Schafen und anderem Vieh, welche im Verlauf von zwei Jahren aufgrund des Opferbetriebes in Assur anfielen: VAT 19193 (Fundnummer Ass. $13058 \mathrm{kl}=$ MARV 2, Text Nr. $19=A L A$ I, M 7, Nr. 175). Es ist gesichert, daß in neuassyrischer Zeit auch Opfertiere Gegenstand des "regelmäßigen Opfers" waren. Daher gibt es guten Grund zu der Vermutung, daß dies auch in mittelassyrischer Zeit so war und lediglich die Archivsegmente der mittelassyrischen Opferverwaltung, in denen Eingang, Verwertung und Verwendung von Opfertieren dokumentiert wurden, nicht auf uns gekommen sind. Sicher ist dies freilich nicht.

9. Siehe z. B. MARV 5, Text Nr. 70:18; MARV 6, Texte Nr. 35:47 und 73:17; MARV 7, Texte Nr. 3:4 und 96:4. 
hohe politische Aufmerksamkeit zu. Dies läßt sich schon daran ermessen, daß sie als vertraglich bindende Vereinbarung geregelt war, die zwischen dem amtierenden obersten Opferverwalter und dem jeweiligen Gouverneur oder Statthalter der einzelnen Provinzen geschlossen wurde. ${ }^{10}$ Jahresabrechnungen in Tabellenform ${ }^{11}$ zeigen überdies, daß im 12. und frühen 11 . vorchristlichen Jahrhundert jeder Verwaltungsdistrikt des Reiches eine genau festgesetzte Menge an Gütern-im wesentlichen Getreide, Honig, Sesam und Früchte-in die Hauptstadt zu liefern hatte. ${ }^{12}$ Bis zu 27 Provinzen sind in solchen Zusammenstellungen genannt. ${ }^{13}$

Da die penibel geführten Auflistungen der gin $\bar{a}^{\prime} u$-Abgaben für eine ganze Reihe von Jahren erhalten blieben, läßt sich ermitteln, daß im Durchschnitt jährlich insgesamt etwa $100 \mathrm{~m}^{3}$ Getreide, $1 \mathrm{~m}^{3}$ Honig, $10 \mathrm{~m}^{3}$ Sesam und $5 \mathrm{~m}^{3}$ Früchte nach Assur geliefert wurden. Pro Provinz entspricht das im Mittel 3,7 $\mathrm{m}^{3}$ Getreide, $37 \mathrm{l}$ Honig, 3701 Sesam und 1851 Früchten. ${ }^{14}$

Diese gewiß nicht übermäßig hohe und daher kaum wirklich zur ökonomischen Last werdende Abgabeverpflichtung bestand, wie den Jahresabrechnungstabellen leicht entnommen werden kann, nur für die der Krone unterstellten Verwaltungsdistrikte, oder anders gesagt: Die für die Speisung des Reichsgottes nach Assur gelieferten Güter kamen ausschließlich aus dem māt Aššur genannten Assyrien, nicht aber aus den Landen tributpflichtiger Vasallen. ${ }^{15}$

Protokolle und Quittungen, die unmittelbar bei der Anlieferung der für das ginā' $u$-Opfer bestimmten Abgaben angefertigt wurden, geben uns einen lebendigen Eindruck des Geschehens. Getreide, ${ }^{16}$ Sesam, Honig ${ }^{17}$ und Früchte ${ }^{18}$ kamen in der

10. Siehe H. Freydank, "Mittelassyrische Opferlisten aus Assur" [wie Anm. 11], 49f.

11. Vgl. z. B. MARV 2, Texte Nr. 21; MARV 5, Texte Nr. 1ff., 58, 64, 67; MARV 6, Texte Nr. 1ff., 82; MARV 7, Texte Nr. 6, 8, 27, 30, 31, 44, 55, 63 und 93; MARV 8, Texte Nr. 24 und 35; MARV 9, Texte Nr. 1, 2, 6, 9 und 80 sowie O. Pedersén, ALA I, 46; siehe H. Freydank, "Mittelassyrische Opferlisten aus Assur", in: Assyrien im Wandel der Zeiten. XXXIXe Rencontre Assyriologique Internationale, Heidelberg, 6.-10. Juli 1992 (ed. H. Waetzoldt und H. Hauptmann; Heidelberg, Heidelberger Orientverlag, 1997), 47-52 und ders., AoF 33 [wie Anm. 14], 219f.

12. In Ausnahmefällen konnten diese Güter durch andere ersetzt werden (siehe z. B. MARV 3, Texte Nr. 35:1ff., 36:1 und40:1ff.; vgl. ferner MARV 7, Text Nr. 62:8).

13. So z. B. in MARV 2, Text Nr. 21.

14. Hierzu siehe O. Pedersén, ALA I, 46 und H. Freydank, "Mittelassyrische Opferlisten aus Assur" [wie Anm. 11]; vgl. auch H. Freydank, "Anmerkungen zu mittelassyrischen Texten 5", AoF 33 (2006), 215-222. Es gilt freilich zu bedenken, daß die aus den Provinzen eingegangenen Beträge im einzelnen bisweilen erheblich höher, manchmal auch geringfügig niedriger sein konnten.

15. Aus der Anzahl der in entsprechenden Urkunden genannten Toponyme wurde mit Recht auf die Ausdehnung des mittelassyrischen Reiches geschlossen, vgl. dazu H. Freydank, "Mittelassyrische Opferlisten aus Assur" [wie Anm. 11], 51 mit Anm. 38.

16. Der weitaus größte Teil der Getreidelieferungen besteht aus Gerste. Daneben sind auch Lieferungen von Emmer (siehe z. B. MARV 5, Text Nr. 23; MARV 7, Texte Nr. 40 und 97) und mirquMehl (siehe z. B. MARV 5, Texte Nr. 27 und 39) bezeugt. Auch "weiße Gerste" (siehe z. B. MARV 9 , Text Nr. 110) und Weizen (siehe z. B. MARV 3, Text Nr. 6 und MARV 6, Text Nr. 73) fanden für die Herstellung der gin $\bar{a} ’ u$-Opfergaben Verwendung.

17. Mit dem Honig kam auch Wachs nach Assur, das ein Vertreter der Schreiber-wohl zur Herstellung von Wachstafeln - entgegennahm. Hierzu siehe MARV 6, Text Nr. 39 und H. Freydank, "»Honig «-Lieferungen für den Gott Assur", AoF 34 (2007), 70-77 mit zahlreichen Korrekturen gegenüber MARV 6.

18. In der Dokumentation der Opferverwalter werden Früchte meist unter dem Sammelbegriff azamru genannt. Nur bisweilen finden sich Angaben darüber, welche Früchte zu den ginä'u-Abgaben zählten. Hierzu gehören Äpfel (siehe z. B. MARV 7, Text Nr. 51:3) und Feigen (siehe z. B. MARV 3, Text Nr. 32:2 und MARV 6, Text Nr. 1:20). 
Regel als Rohstoffe in noch unverarbeiteter Form ${ }^{19}$ aus den Provinzen nach Assur und wurden erst danach in den Werkstätten und Küchen des Tempels zu Speisen und Getränken weiterverarbeitet, welche für die tägliche Grundversorgung des Reichsgottes und seines göttlichen Gefolges bestimmt waren.

Zumeist erreichten die Naturalienlieferungen die assyrische Hauptstadt auf dem Wasserweg. ${ }^{20}$

Manche Urkunden aus dem Archiv der Opferverwalter erweisen sich als vor Ort angefertigte Lieferungsprotokolle. Schon deren Schriftbild läßt den Vorgang der Übergabe der Güter in die Verantwortung des Assur-Tempels wieder lebendig werden. Die Lieferanten hatten nämlich—so zeigen es diese Texte-die Nahrungsmittel und namentlich das Getreide vor Vertretern der Opferverwaltung darzumessen. Zu diesem Zweck wurde unmittelbar nach der Ankunft in Assur-vielleicht bereits am Hafenkai-das stets in »Eselslasten (emāru) " gemessene Getreide in Einheiten von 'halben Eselslasten' übergeben. Ein Tempelbeamter registrierte dabei den Empfang einer jeden, wohl in Gestalt von Getreidesäcken ${ }^{21}$ abgegebenen 'halben Eselslast', indem er dies mit einem stehenden Keil in einer "Strichliste ${ }^{22}$ vermerkte und dabei nach und nach jeweils kleine, aus zehn Keilen bestehende »Päckchen « entstehen ließ (Abb. 4 und Abb. 5). ${ }^{23}$

In anderen Dokumenten der Opferverwalter wurde wohl ebenfalls gleich nach der Anlieferung festgehalten, daß die erwartete Sendung vollständig und ordnungsgemäß übergeben worden waren. Dabei notierte man auch die Namen der Schiffer, die die Güter nach Assur transportiert hatten. ${ }^{24}$ Waren transportbedingt Verluste zu beklagen-etwa wenn Wasser in das Frachtschiff geschwappt und Getreide naß geworden war-mußten die Lieferanten noch vor Ort einen entsprechenden Schuldschein zeichnen. ${ }^{25}$ Auch wenn eine Lieferung grundsätzlich geringer ausgefallen war, als dies in Assur erwartet wurde, stellte man dem für die säumige Provinz

19. Statt Sesam wurde bisweilen aus Sesam gepreßtes Öl geliefert (siehe z. B. MARV 5, Texte Nr. 27, 55; siehe ferner MARV 3, Text Nr. 9). Im Normalfall wurde jedoch der aus ginā'u-Abgaben stammende Sesam in den Werkstätten des Assur-Tempels zu Öl weiterverarbeitet (siehe z. B. MARV 7, Texte Nr. 27, 78 und 79; MARV 8, Text Nr. 60).

20. Siehe z. B. MARV 6, Texte Nr. 3 und 29; MARV 7, Text Nr. 8; MARV 8, Text Nr. 27; MARV 9 , Texte Nr. 15 und 95. Vgl. aber auch MARV 6, Text Nr. 13 als Beispiel für die aus der Provinz Kilizu entrichtete Abgabe von 4 Eselslasten Sesam, die auf dem Landweg nach Assur gelangten.

21. Die Einheit der 'halben Eselslast' ergibt sich nahezu von selbst, wenn man davon ausgeht, daß die Getreidemenge, die man einem Esel aufbürden kann (etwa $100 \mathrm{~kg}$ ), zum Transport regelmäßig auf zwei Satteltaschen verteilt wurde und dabei eine solche Satteltasche eine nach und nach normierte Verpackungsgröße bildete. In den hier besprochenen Urkunden sind freilich diese Satteltaschen oder Säcke nie explizit genannt.

22. Beispiele für Dokumente der Opferverwalter, die solche vor Ort erstellten Strichlisten enthalten sind: $M A R V$ 5, Text Nr. 57; MARV 7, Texte Nr. 22, 46 (siehe Abb. 4a-b), 61, 83; MARV 8, Texte Nr. 13, 27, 30; MARV 9, Text Nr. 16 sowie das noch unveröffentlichte Dokument VAT 15426 (siehe Abb. 5a-b), in dem Lieferungen aus der Provinz Katmuhu verzeichnet sind. Alle Tafeln sind so organisiert, daß-falls mehr als 100 halbe Eselslasten gezählt wurden-in der ersten Zeile der 'Strichlisten' stets insgesamt 100 Keil-'Striche' stehen. Nur in MARV 7, Text Nr. 46 (Abb. 4) wurden größere Einheiten (?) gesondert auf der Tafelrückseite mit einem runden Griffeleindruck vermerkt. Der Zusammenhang mit der 'Strichliste' auf der Tafelvorderseite bleibt unklar. Möglicherweise entspricht ein runder Griffeleindruck auf der Rückseite jeweils einem auf der Vorderseite mit einzelnen Keilen zusammengesetzten "Paket« von 10 halben Eselslasten.

23. Siehe dazu auch H. Freydank, MARV 5, 12 zu Text Nr. 57.

24. Als Beispiel hierfür sei stellvertretend für zahlreiche weitere Urkunden MARV 9, Text Nr. 95 genannt.

25. Siehe z. B. MARV 1, Text Nr. 66 (=MARV 6, Text Nr. 28!); MARV 3, Texte Nr. 14, 27, 38; MARV 6, Text Nr. 42. 

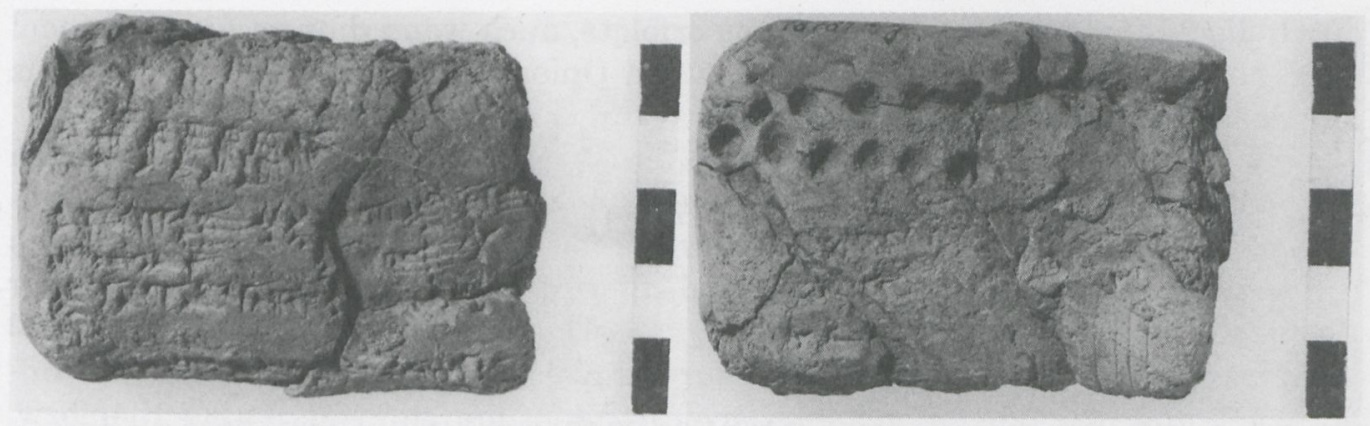

Abb. 4. VAT 20005, Vs. und Rs. (= MARV 7, Text Nr. 46). Protokoll der Lieferung von 166 halben Eselslasten Getreide für den Assur-Tempel.

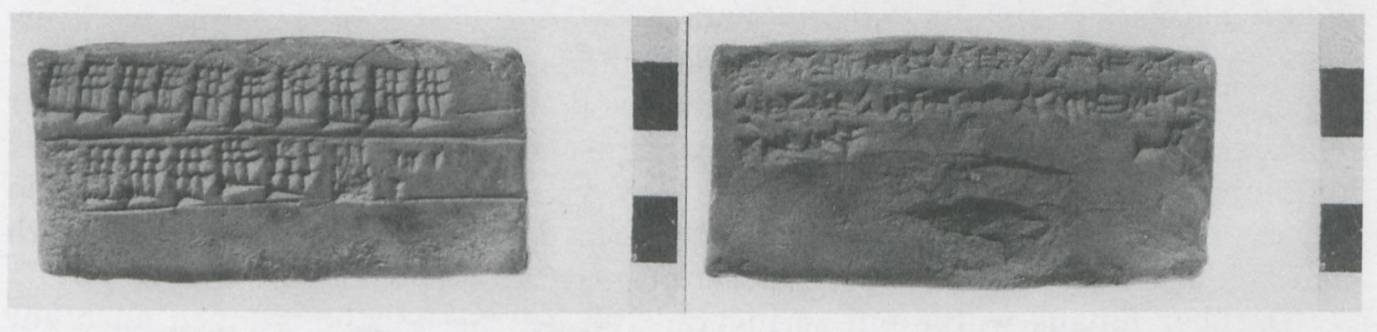

Abb. 5. VAT 15426, Vs. und Rs. (unveröffentlicht). Protokoll der Lieferung von 174 halben Eselslasten Getreide für den Assur-Tempel.

verantwortlichen Gouverneur, Statthalter oder 'Bürgermeister' einen Schuldschein aus, in dem in der Regel der Zeitpunkt genannt ist, bis zu dem die Ausstände spätestens beglichen sein sollten. ${ }^{26}$ In nicht wenigen Urkunden wurde dabei der auch im Assur-Tempel verehrte, "Sonnensohn" genannte Stiermensch kusarikku $\left({ }^{\mathrm{d}} \mathrm{GU}_{4^{-}}\right.$ DUMU-dUTU) als Zeuge angerufen. ${ }^{27}$ In manchen Fällen drohte man gar mit der Erhebung von Verzugszinsen, falls die Rückzahlung der Schuld nicht in dem vereinbarten Zeitraum erfolgte. ${ }^{28}$ Über die dennoch ausstehenden Lieferungen wurde offenbar mit großer Sorgfalt buchgeführt. ${ }^{29}$ Kein einziges Dokument aus den Archiven der Opferverwalter läßt erkennen, daß die Begleichung solcher Verbindlichkeiten

26. Siehe z. B. MARV 3, Texte Nr. 28, 30, 31, 35, 44, 55; MARV 5, Texte Nr. 41 und 44; MARV 7, Text Nr. 76.

27. So z. B. in: MARV 3, Texte Nr. 14:16, 20:16, 30:19, 31:15, 32:18, 38:15, 50:19, 51:13; MARV 5, Text Nr. 41:19; MARV 7, Text Nr. 76:19'. Siehe dazu auch H. Freydank, Beiträge zur mittelassyrischen Chronologie und Geschichte (Berlin: Akademie-Verlag, 1991), 67 und 70 mit Anm. 183 sowie ders., AoF 19 (1992), 301 zu Z. 16. Zu dem im Assur-Tempel verehrten Stiermenschen kusarikku siehe A. R. George, Babylonian Topographical Texts (Leuven: Dep. Oriëntalistiek [u.a.], 1992), 188:37' und 190, 25' sowie ferner die von R. Borger in Mesopotamisches Zeichenlexikon, 2. revidierte und aktualisierte Auflage (Münster: Ugarit-Verlag, 2010), 337 zusammengestellte Literatur. Es ist sicher kein Zufall, $\mathrm{da} \beta$ in mittelassyrischer Zeit ein hochstehender Brauer des Assur-Tempels, der maßgeblich mit der Bereitstellung des ginā' $u$-Opfers betraut gewesen sein muß, "für sein Leben" dem kusarikku ein bedeutendes Weihegeschenk zukommen ließ (Zu dem Hortfund auf dem großen Vorhof des Tempels siehe O. Pedersén, ALA I, 53 und E. Klengel-Brandt, J. Marzahn, "Ein Hortfund mit Kreuzen aus Assur", BagM 28 [1997], 209-238 und Tf. 16-27).

28. So in MARV 3, Texte Nr. 14 (falls die Schuld nicht innerhalb von 40 Tagen beglichen sein sollte) und 50 (falls die Schuld nicht innerhalb eines Monats beglichen sein sollte).

29. Siehe etwa MARV 6, Text Nr. 42 mit einer langen Liste solcher Schuldbeträge. Sie ist als "zweite noch nicht abgeschlossene Tafel” (Z. 44) bezeichnet. 
durch die Zahlung eines Silberbetrages erfolgte, auch wenn dies im freien Handel durchaus vorstellbar gewesen wäre. Bei den Opferverwaltern des Assur-Tempels gingen ausschließlich Naturalien ein.

Weitere Dokumente lassen erkennen, daß — schon bei der Anlieferung-neben den Opferverwaltern auch diejenigen zugegen waren, die die eingehenden Rohwaren im Auftrag des Tempels weiterverarbeiten sollten. Beachtliche Mengen des angelieferten Getreides wurden nämlich sogleich, vielleicht sogar noch an der Bootsanlegestelle zu Füßen des Tempels, an Bäcker (alahhin $\bar{u})$ und Brauer (sirāšū) weitergegeben, die im Dienste des Assur-Tempels standen. ${ }^{30}$ Sie hatten die Aufgabe, die angelieferte Gerste weiterzuverarbeiten, um dann Malz und Bier bzw. Getreidespeisen, Brot und Backwaren herzustellen, die dem Assur und seinem göttlichen Hofstaat dargebracht werden sollten. ${ }^{31}$ Den Ölpressern des Tempels wurden Sesamlieferungen zur Weiterverarbeitung ausgehändigt. ${ }^{32}$

All dies dokumentieren entsprechende Empfangsquittungen und auch werkvertragsähnliche schriftliche Vereinbarungen, in denen die ausgegebenen Nahrungsmittel, die Art des herzustellenden Produktes und der vorgesehene Zeitpunkt der Fertigstellung bzw. der Tag der Darbringung als Opfer genau festgelegt sind. ${ }^{33}$ Bei Erfüllung des Auftrages entwerteten die Opferverwalter die Urkunden, aus denen hervorging, daß aus ihrer Lagerhaltung Güter an die betreffenden, stets namentlich genannten Brauer, Müller und Ölpresser ausgegeben worden waren. ${ }^{34}$

Darüber hinaus wurde ein geringerer Teil der Getreidelieferungen auch zur Verpflegung derjenigen Arbeiter eingesetzt, die in den Wirtschaftsräumen des Tempels Bier brauten und Öl, Brot, Kuchen und andere Getreideprodukte herstellten. ${ }^{35}$

Ein weiterer Teil des angelieferten Getreides diente den Opferverwaltern dazu, denjenigen Darlehen zu gewähren, die ihre Lieferverpflichtungen nicht erfüllt hatten, so daß dennoch die für den Gott bestimmte Lieferung in ihrem Namen eingesetzt werden konnte. ${ }^{36}$ Erst bei der Begleichung der Ausstände wurden die ausgestellten und archivierten Schuldscheine durch das Durchstreichen der Urkunde sichtbar entwertet (Abb. 6). ${ }^{37}$

Das Ausbleiben von Lieferungen aus den assyrischen Landen sollte jedoch auf gar keinen Fall zu einer Störung des Opferbetriebes führen. Als nämlich tatsächlich einmal ein solcher Engpaß entstand, und so der "Abbruch des regelmäßigen

30. Siehe z. B. MARV 1, Texte Nr. 11, 25; MARV 5, Texte Nr. 16, 21, 26, 28, 29, 49, 50, 65; MARV 6, Texte Nr. 19, 24, 69, 73, 81; MARV 7, Text Nr. 91; MARV 8, Texte Nr. 46, 48; MARV 9, Texte Nr. 14, $31,96,110,112$ und passim.

31. Siehe z. B. MARV 5, Texte Nr. 26 (Herstellung von Malz) und 70; MARV 6, Text Nr. 73; MARV 9, Text Nr. 110.

32. Siehe z. B. MARV 7, Texte Nr. 32, 78 und 79; siehe auch MARV 8, Text Nr. 60.

33. Siehe z. B. MARV 6, Texte Nr. 20, 33, 36 und 40. In zwei mir bekannten Urkunden ist ein bēt ginä'e genannt (MARV 3 Nr. 76 [= MARV 6, Text Nr. 8!] und MARV 6, Text Nr. 12). Möglicherweise ist bēt gină'e die Bezeichnung jener Bereiche innerhalb des Tempelzingels, in denen die für das gină $u$ Opfer verwendeten Speisen hergestellt oder aufbewahrt wurden.

34. Die solche Ausstände belegenden Urkunden wurden ungültig gemacht, indem man meist über Vorder- und Rückseite ein diagonal verlaufendes Kreuz (crux decussata) ritzte. Siehe z. B. MARV 5, Text Nr. 21, MARV 6, Text Nr. 24; MARV 7, Texte Nr. 2 und 32; MARV 9, Text Nr. 116.

35. Siehe z. B. MARV 1, Text Nr. 49; MARV 5, Texte Nr. 8, 17; MARV 7, Texte Nr. 4, 43; MARV 9, Text Nr. 23.

36. Hierzu siehe Freydank, AoF 19, 278-281. Belege hierfür lassen sich passim in den hier beschriebenen Archiven der Opferverwalter finden.

37. Zu der in Abb. 6 gezeigten Tafel VAT $19986=$ MARV 7, Text Nr. 76 siehe auch J. Llop, OrNS 77 (2008), $180 \mathrm{f}$. 


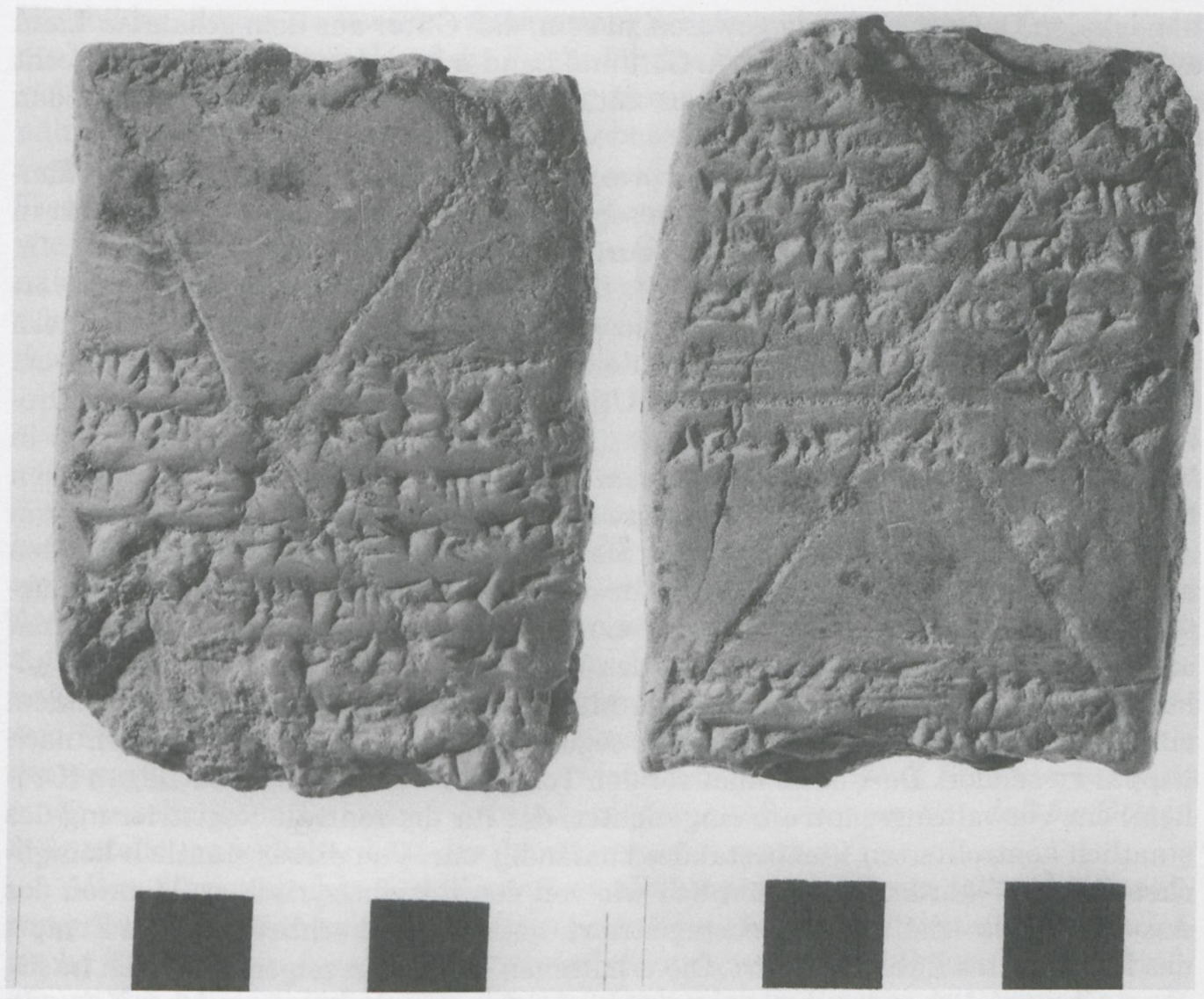

Abb. 6. VAT 19986 (= MARV 7, Text Nr. 76). Eine durch Durchstreichen ungültig gemachte Schuldurkunde, in der ausstehende Lieferungen für das ginā'u-Opfer aufgeführt sind

Opfers für den Assur-Tempel (batiqti ša ginā’e ša bēt Aššur)" drohte, setzte der "Opferaufseher" (ša muhhi ginā’e) Aba-lā-īde-wie uns die als MARV 3, Text Nr. 34 veröffentlichte Urkunde zeigt ${ }^{38}$ - sein eigenes Vermögen (ša raminīšu) dafür ein, daß die Götter des Hauptheiligtums des Reiches weiterhin mit Speis und Trank versorgt wurden. "Damit dies nicht untergehe (ana lā mašăe),", und er das dem Tempel überlassene Darlehen zurückfordern konnte, ließ Aba-lā-īde sich seine Hilfeleistung freilich quittieren.

Die tägliche Versorgung der Götter des Assur-Tempels hätte wohl ohne weiteres auch aus königlichen Domänen oder aus Tempelländereien gedeckt oder gänzlich aus dem Umland der Hauptstadt bestritten werden können. Indes zeigt die hier nur kurz vorgestellte, gewissenhaft geführte Dokumentation der Opferverwalter des Assur-Tempels deutlich, daß dies gar nicht beabsichtigt war. Denn man legte offensichtlich den größten Wert darauf, daß die Grundversorgung des Gottes von allen Teilen des assyrischen Reiches gemeinsam geleistet wurde. Weit wichtiger als die Notwendigkeit, die beachtliche Menge von Naturalien für das regelmäßige Opfer zusammenzutragen, scheint der Gedanke der im Auftrag von König und Tempel

38. Vgl. die Bearbeitung: H. Freydank, AoF 19 (1992), 285, Text Nr. 2. 
handelnden Opferverwaltung gewesen zu sein, daß Güter aus dem gesamten Land auf den Tisch des Gottes gelangten. Gott und Land tragen so nämlich ganz zu Recht den gleichen Namen: Das Land Assur mit allen seinen einzelnen Teilen nährt den Gott, der das Land selbst verkörpert.

Dieser die mittelassyrische Opferpraxis charakterisierende Gedanke ist offenbar sehr alt und besitzt eine lange mesopotamische Vorgeschichte, die sich bis in das dritte vorchristliche Jahrtausend zurückverfolgen läßt. Denn schon im 21. Jh. v. Chr., in der Zeit des Reiches der III. Dynastie von Ur, findet er in Abgabeverpflichtungen seinen Ausdruck, die - ganz so wie in der späteren Zeit-Statthaltern und Gouverneuren der einzelnen zum Reich gehörigen Provinzen auferlegt waren. Einem Corpus von mehreren Hundert Urkunden aus Puzriš-Dagan (modern: Drehem) können wir entnehmen, woher das Fleisch kam, welches man Enlil, dem in Nippur verehrten Götterkönig, in seinem Tempel Ekur im Rahmen des täglichen Mahls vorsetzte. ${ }^{39}$ Das hierfür benötigte Schlachtvieh stammte keineswegs allein aus den großen Herdenbeständen des Staates und der Tempel. Vielmehr wurden aus allen Regionen des Reiches Tiere für das Opfer geliefert. Jahr für Jahr schickten Statthalter und Gouverneure der einzelnen Provinzen ein gemästetes Schaf oder ein Ziegenböckchen als Gabe für den Reichsgott Enlil, ohne die zunächst vielleicht unverhältnismäßig erscheinende Mühe zu scheuen, einen Boten mit einem einzelnen Tier über Entfernungen von sogar mehreren Hundert Kilometern nach Nippur zu senden. Dort hatte man vor den Toren der Stadt mit Puzriš-Dagan (Drehem) ein Verwaltungszentrum eingerichtet, das für die zentrale Registrierung des staatlich kontrollierten Viehbestandes zuständig war. Von dieser staatlich-königlichen Behörde wurden-ganz ähnlich wie von den mittelassyrischen Beamten des Assur-Tempels-die Opfergaben registriert, quittiert und schließlich dem Tempel des Reichsgottes Enlil zugeführt. Die erhaltenen Urkunden zeigen, daß auch im 21. Jh. v. Chr. peinlich genau verbucht wurde, welcher Statthalter zu welcher Zeit sein jährlich bereitzustellendes Opfertier nach Nippur hatte bringen lassen.

Den uralten Gedanken, daß alle Landesteile ihren Gott gemeinsam ernähren sollten, finden wir auch in Quellen der neuassyrischen Zeit wieder, diesmal aus der Perspektive eines allumfassenden Weltherrschaftsanspruchs ins monumental Kosmische überhöht. ${ }^{40}$ Eine Königsinschrift des Asarhaddon (680-669 v. Chr.), in der dieser die Feierlichkeiten schildert, die er anläßlich des Richtfestes für den von ihm renovierten Tempel des Reichsgottes Assur angeordnet hatte, heißt es: "Ich schlachtete gemästete Stiere, schächtete Edelschafe und köpfte Vögel des Himmels und Fische der Wassertiefen ohne Zahl. Ausbeute des Meeres und Ertrag des Gebirges häufte ich vor (den Göttern des Assur-Tempels) auf. ... Abgaben (igisê) aus (allen) Ortschaften, schwerwiegendes Begrüßungsgeschenk ließ ich sie empfangen und machte ihnen zahlreiche Geschenke." 41

Die Tiere lieferten hier nicht allein die Nahrung für den Gott, sondern sie repräsentierten auch und vor allem die drei kosmischen Bereiche des altorientalischen

39. Vgl. die von W. Sallaberger vorgelegte grundlegende Wertung dieses Textfundes: W. Sallaberger, "Schlachtvieh aus Puzrisch-Dagan. Zur Bedeutung dieses königlichen Archivs", Jaarbericht Ex Oriente Lux 38 (2003-2004), 45-62.

40. Es ist freilich nicht unwahrscheinlich, daß die "kosmische « Konzeption des Opfers vor Assur, wie wir sie in der im folgenden zitierten Inschrift des Asarhaddon fassen können, bereits in mittelassyrischer Zeit existierte.

41. Siehe R. Borger, Die Inschriften Asarhaddons, Königs von Assyrien, AfO Beiheft 9 (Graz: Selbstverlag des Herausgebers, 1956), 5 (§ 2, Ass. A, VI:37-VII:12). 
Weltbildes, denen sie jeweils entstammen: Schafe und Stiere stehen für die Erde; die Vögel für den Himmel und die Fische für den Süßwasser-Ozean (apsû), über den sich die Erde wölbt. Mit der Darbringung dieser Opfertiere wird der höchste Gott ernährt, indem er getragen wird von der Lebenskraft des gesamten Kosmos in seiner vertikalen Ordnung: von Himmel, Erde und Süßwasser-Ozean (apsû). Da zu den Tieren aller Art, die König und Tempel bereitgestellt haben mochten, auch noch, wie unser Text sagt, "Abgaben aus (allen) Ortschaften" kamen, liegt der Gedanke nahe, daß der gesamte Raum und die gesamte Gemeinschaft der (zivilisierten) Menschen, also gewissermaßen das "All« dem Gott seinen Tribut zu bringen hatte, um ihn gemeinschaftlich zu ernähren.

Die hier insbesondere für die Zeit des ausgehenden 2. Jt. v. Chr. beschriebene assyrische Opferpraxis ist nicht zuletzt von dem theologisch motivierten Streben getragen, jenem in den Schöpfungsmythen immer wieder artikulierten göttlichen Auftrag an die Menschheit nachzukommen, in dem nach mesopotamischer Vorstellung die Daseinsberechtigung des Menschen liegt. Einhellig berichten nämlich sowohl der uralte sumerische Mythos Enki und Ninmah, als auch die altbabylonische Atramhasīs-Erzählung und das im späten zweiten vorchristlichen Jahrtausend entstandene babylonische Weltschöpfungsepos Enūma elišs, daß der Mensch allein deshalb erschaffen worden sei, um die Götter mit Speis und Trank zu versorgen. Hege und Pflege der Götter ist diesen Mythen zufolge-aller Geschäftigkeit zum Trotzdie eigentliche, die wahre Aufgabe des Menschen, der als Dank für seine Existenz einen ansehnlichen Teil seiner Arbeitskraft dafür aufzubringen hat, daß die Götter, freigestellt von jeglicher Last der Arbeit, umsorgt werden. ${ }^{42}$

Allen hierarchischen Gesellschaftsvorstellungen zum Trotz sollte die Ernährung des Gottes aber keinesfalls allein beim König liegen, obgleich sich doch sumerische Stadtfürsten des dritten vorchristlichen Jahrtausends ebenso wie die Großkönige des neuassyrischen und des neubabylonischen Reiches mit Beinamen wie "Versorger der Gottheit N. N." oder "Versorger des Tempels N. N." schmückten. ${ }^{43}$ Dem König Assyriens kam es zwar zu, die Gaben jeder einzelnen Provinz erfassen, verarbeiten und dem Gott zuführen zu lassen, bereichert um hinzugefügte eigene Gaben. In der Funktion des irdischen Statthalters seines Gottes stellte er so sicher, $\mathrm{da} ß$ der an die Menschheit ergangene göttliche Auftrag erfüllt wurde. In dem assyrischen Opferwesen erhielt aber dennoch der in den Schöpfungsmythen besonders hervorgehobene Gedanke, daß es die Arbeit (aller) Menschen sei, die die Versorgung der Götter sicherzustellen habe, besonders großes Gewicht. ${ }^{44}$

Eine Urkunde aus dem mittelassyrischen Archiv der Opferverwalter führt uns dies deutlich vor Augen. ${ }^{45}$ Das Dokument enthält eine Aufstellung von insgesamt 49 »Getreidemahlern des Assur-Tempels« ( $t \bar{e} ’ i n \bar{u}$ ša bēt Aššur ${ }^{46}$ ), denen die Aufgabe

42. Siehe hierzu S. M. Maul, "Den Gott ernähren. Überlegungen zum regelmäßigen Opfer in altorientalischen Tempeln", in: E. Stavrianopoulou, A. Michaels, C. Ambos (Hrsg.), Transformations in Sacrificial Practices. From Antiquity to Modern Times, (Berlin; Münster: LIT, 2008), 75-86 [besonders 76-80].

43. Belege bei M.-J. Seux, Épithètes royales akkadiennes et sumériennes (Paris: Letouzey et Ané, 1967) unter den Stichwörtern zäninu und ú-a.

44. Die uns erhalten gebliebenen Dokumentationen reichen freilich bei weitem nicht aus, um zu entscheiden, ob hier ein Phänomen beschrieben wird, das die assyrische Opferpraxis von der babylonischen oder der des 3. vorchristlichen Jahrtausends unterscheidet.

45. H. Freydank, MARV 5, Text Nr. 60 (VAT 20921). Der Text ist dort lediglich als Keilschriftautographie veröffentlicht.

46. Die têe'inu oder $t \bar{a}^{\prime}$ inu (nicht in den Wörterbüchern gebucht; siehe aber MARV 7, Text Nr. 4:26') genannten Handwerker sind in den Dokumenten der Opferverwalter aus Assur nur selten belegt. Die 
zukam, die aus den Provinzen des Reiches eingehende, für die Speisung des Assur bestimmte Gerste vor Ort im Assur-Tempel zu mahlen. In der Urkunde sind zwar nicht die Namen dieser Leute, dafür aber auffälligerweise die Herkunft einer jeden Arbeitskraft genannt. Obgleich in der Hauptstadt und deren Umland sicherlich ohne weiteres genügend Müller hätten rekrutiert werden können, um das eingehende Getreide für die Weiterverarbeitung aufzubereiten, kamen für diese Arbeit nur sehr wenige Menschen aus Assur zum Einsatz. ${ }^{47}$ Neben diesen hatten an der Verrichtung des Getreidemahlens nämlich weit mehr als vierzig andere Personen Anteil, die wohl nicht zufällig aus insgesamt 23 weiteren Verwaltungsbezirken und Provinzen des assyrischen Königreiches stammten. Hinzu kamen noch zwei gesondert aufgeführte "Getreidemahler,${ }^{48}$ die nicht von den unterschiedlichen Territorien des Reiches, sondern - wie man vermuten muß - von einer Person oder aber einer Institution gestellt wurden. Leider blieb die Angabe, die hier Klarheit hätte bringen können, in der Urkunde (MARV 5, Text Nr.60:29) nicht erhalten. Man wird aber mit einigem Recht davon ausgehen können, daß es entweder der Palast oder aber der Haupttempel des Gottes Assur war, in dessen Namen man diese Arbeitskräfte der 'internationalen Gemeinschaft' von Müllern noch hinzugesellte.

Der alleinige Zweck der Urkunde, zu der sich bislang lediglich ein einziges Vergleichsstück nachweisen läßt, ${ }^{49}$ kann nur darin gelegen haben zu dokumentieren, daß das gesamte Land, vertreten durch sein Territorien, nicht allein die Opfermaterie hervorgebracht hatte, sondern darüber hinaus auch-institutionell abgesichert-an der Bereitung der Götterspeisen Anteil haben würde! Wir wissen nicht, ob die aus den Provinzen entsandten Männer dauerhaft an der Opferbereitung beteiligt waren, oder ob sie bei bestimmten Gelegenheiten eigens für das Mahlen des Opfergetreides anreisten. Auch ist leider unbekannt, welcher sozialen Schicht sie angehörten. Wurden wirklich einfache Arbeiter in die Haupstadt geschickt, oder könnten es auch Honoratioren gewesen sein, die es als erstrebenswerte Ehre, ja als Gottesdienst empfanden, an der Speisung des Reichsgottes aktiv mitwirken zu dürfen?-Auch wenn entsprechende Erwähnungen oder gar Ritualbeschreibungen fehlen, kann man nicht ausschließen, daß ausgewählte Vertreter aller Landesteile in regelmäßigen Abständen zusammenkamen, um in einem großartig inszenierten performativen Akt, gemeinsam dem höchsten Gott das Opfer zu bereiten und so auch sichtbar und erlebbar die Gemeinschaft einer communio assyria zu feiern.

Wie dem auch sei, der in den Schöpfungsmythen formulierte Anspruch an die Menschen, daß die Arbeit aller die Götter ernähren möge, wurde in Assyrien auf diese Weise bis in das Wörtlichste hinein umgesetzt. An der regelmäßigen Versorgung des höchsten assyrischen Gottes hatten nicht nur die Territorien und Provinzen des Reiches samt ihrer Bevölkerung teil. Die Gemeinschaft der Opferbringer war außer von dieser 'horizontalen' Komponente auch von einer vertikalen geprägt. Die Urkunde MARV 8, Text Nr. 68 lehrt nämlich, daß schon in mittelassyrischer Zeit—so wie wir es auch aus den späteren Jahrhunderten kennen ${ }^{50}$-neben den Vertretern der einzelnen Landesteile auch die hohen, in der Hauptstadt angesiedel-

Arbeit des Getreidemahlens scheint sonst in den Bereich der alahhinū (siehe z. B. KAJ 318:6) bzw. der sāmidū zu gehören.

47. Aus der Stadt Assur waren zwei, maximal drei "Getreidemahler« für diese Arbeiten eingesetzt (MARV 5, Text Nr. 60:12). Hinzu kamen ein bis zwei weitere aus dem Libbi-äli genannten Palast- und Tempelbezirk der Stadt (MARV 5, Text Nr. 60:16).

48. MARV 5, Text Nr. 60:29.

49. MARV 6, Text Nr. 64 (VAT 20946).

50. Siehe Anm. 54. 
ten Würdenträger des Reiches Naturalien für die Bereitung des gin $\bar{a}^{\jmath} u$ genannten, regelmäßigen Opfers bereitstellten.

Durch ihre Arbeit brachten so in der späten mittelassyrischen Zeit neben dem König Gouverneure, Beamte und hohe Würdenträger, Handwerker, Bauern und wohl auch Hirten und Viehzüchter ${ }^{51}$ die für den Gott bestimmten, täglich dargebrachten Speisen hervor. Diese konnten mit Fug und Recht als eine Gabe betrachtet werden, die eine alle Gesellschaftsschichten umfassende Gemeinschaft in ihrer gesamten territorialen Ausdehnung hervorgebracht hatte. Die Identitätsstiftende Kraft, die einer solchen Vorstellung des Opfers mit sich bringt, sollte nicht unterschätzt werden! Aus Herren und Untertanen wird so im performativen Akt des Opferns ein Gottesvolk. Im Falle Assyriens, in dem der Name des Gottes Assur auch das Land und dessen Bewohner bezeichnet, wird dies in besonderer Weise deutlich deutlich. $^{52}$

Bezeichnenderweise wurden in dem stark expandierenden neuassyrischen Reich neu eingegliederte Provinzen dazu verpflichtet, sich an der regelmäßigen Speisung des Reichsgottes zu beteiligen. So versuchte König Asarhaddon (680-669 v. Chr.), das eroberte Ägypten nicht nur unter einem Gouverneur in das assyrische Herrschaftsgebiet, das Land Assur, zu zwingen, sondern er erlegte ihm gleichzeitig, wie wir aus seinen Inschriften erfahren, die Pflicht auf, "bis in die Ewigkeit regelmäBige Opfer für Assur und die großen Götter" ${ }^{53}$ zu entrichten. Das auf solche Weise erzwungene regelmäßige Opfer nötigte dem Besiegten, neben allem anderen auch noch die bis weit in die Transzendenz reichenden Verpflichtungen gegenüber einer fremden, ursprünglich dem Feind zugeordneten Gottheit ab, um damit göttliches Wohlwollen für jene zu erwirken, die das eigene Staatswesen entmachtet hatten. . .

Wenn nun, wie im assyrischen Opferwesen, der großen Gemeinschaft von König und den ihm Unterstellten die Aufgabe zukam, den Reichsgott zu nähren und so nachhaltig göttliche Gunst sicherzustellen, bedeutet das im Umkehrschluß, daß eine Verweigerung der Speisegabe einem sich der »Ernährungsgemeinschaft« Entziehen und damit dem Leugnen gleichkommt, zu den Menschen zu zählen, für die der König vor dem Reichsgott Verantwortung hat. Eine Verweigerung der Speisegabe für den Reichsgott Assur unterscheidet sich daher kaum von einer Haltung, die Aufstand als unumgänglich betrachtet! Mit einem Mal wird klar, warum in dem mittelassyrischen Archiv der Opferverwalter so peinlich genau darüber Buch geführt wurde, wer seine Abgabe nicht erbracht hatte, und warum die entsprechenden, aus neuassyrischer Zeit auf uns gekommenen Urkunden ${ }^{54}$ (worüber sich die Herausgeber wundern ${ }^{55}$ ) nicht im Assur-Tempel sondern im Königspalast zu Ninive aufbewahrt wurden.

Eine fehlende, nicht eingegangene Opfergabe konnte man zwar auch in neuassyrischer Zeit leicht verschmerzen, wie folgender, in Ninive gefundener Brief eines Assur-Priesters an den König deutlich zeigt:

51. Vgl. hierzu Anm. 8.

52. Hierzu siehe auch H. D. Galter, "Gott, König, Vaterland. Orthographisches zu Assur in altassyrischer Zeit”, WZKM 86 (1996) [= Festschrift H. Hirsch], 127-141.

53. Siehe R. Borger, Die Inschriften Asarhaddons [wie Anm. 41], 99 (§ 65, Mnm. A [Zinçirli-Stele], Rs. 48-49).

54. F. M. Fales, J. N. Postgate, Imperial Administrative Records. Part I: Palace and Temple Administration, State Archives of Assyria 7 (Helsinki: Helsinki University Press, 1992), 182-203 und G. van Driel, The Cult of Aššur (Assen: van Gorcum, 1969), 206-220.

55. F. M. Fales, J. N. Postgate, Imperial Administrative Records [wie Anm. 54], XXXV: "Why these tablets should have been found at Nineveh remains obscure, but part of the explanation must be that the contributions come from members of the royal household." 
Der heutige 5 . Kanunu ${ }^{56}$ ist von der Stadt Talmusu zu bestreiten. Nichts wurde geliefert. Niemand kam her. Ich habe (dennoch) für das Leben des Königs, meines Herrn, alle Opfer vor Assur [und den Göttern des] Königs, meines Herrn, dargebracht. ${ }^{57}$

Das fehlende Opfergut konnte ohne weiteres aus dem Vermögen des Assur-Tempel erbracht werden. Aber darum ging es nicht. Der König selbst war an den Namen derjenigen die säumig blieben, höchst interessiert und forderte die Leitung des Assur-Tempels auf, ihm entsprechende Informationen zuzusenden. ${ }^{58}$ Denn die verdeckte Renitenz, die das Nichtliefern der erwarteten Gaben darstellte, war nicht hinzunehmen, und wurde, wie ein anderer Brief aus den Staatsarchiven der neuassyrischen Könige des 7. vorchristlichen Jahrhunderts deutlich zeigt, geahndet:

[An den König], meinen Herrn: [Dein Diener D]adî. [Heil], dem König, meinem Herrn. Mögen Nabû und Marduk den König, meinen Herrn, segnen!

Zwei Rinder und 20 Schafe, Opfergaben des Königs, die die Stadt Diquqina zu erbringen hat, sind nicht geliefert worden. Der König, mein Herr, möge dieser Angelegenheit nachgehen. (...) Es sind nun $[x]$ Jahre, daß sie nicht geliefert haben. Die haben das eingestellt. Der König, mein Herr, sollte seine Soldaten [dort hinschicken ]." 59

Wir beobachten hier, wie die »Ernährungsgemeinschaft des Assur« sich auf dem Weg befindet, einer Art Staatsidentität zu entfalten: Derjenige kann sich Assyrer nennen, der in der umfassenden Gemeinschaft der sozialen Schichten, der Städte und Provinzen an der Versorgung jener Gottheit teilhat, die den Namen des Landes Assur trägt und deren Unterhalt der assyrische König zu gewährleisten hat. Der Weg von einer Opfergemeinschaft zu einer gewissermaßen übernationalen Gemeinschaft des assyrischen Volkes wird hier beschritten.

Die "Reste" der dem Gott vorgesetzten Gaben, wurden nach der Speisung des Gottes (zumindest in neuassyrischer Zeit) abgeräumt und an den König, an hochstehende Palastangehörige an Provinzstatthalter, Priester und Tempelpersonal verteilt. ${ }^{60}$ Wer diese Reste ißt, so ist es ausdrücklich in einem neuassyrischen Brief an den König gesagt, "der wird leben". ${ }^{61}$ Aus der Gemeinschaft der Gottesernährer wird so auch eine Gemeinschaft, die mit Götterspeise nicht nur den Gott, sondern auch ihren König und sich selbst ernährt.

Der mit einer solchen Götterspeisung verbundene Gedanke einer Gemeinschaft, die die Menschen untereinander ebenso verbindet wie das Gottesvolk mit seinem göttlichen Herrn, wird zumindest der Gemeinschaft der Willigen Identität und Festigkeit verliehen und so schon in mittelassyrischer Zeit dem assyrischen Reich in nicht unerheblichem Maße zu innerer Stabilität verholfen haben.

56. Der Kanunu ist der zehnte Monat des Jahres. Er entspricht etwa dem Zeitraum Dezember/ Januar.

57. S. W. Cole, P. Machinist, Letters from Priests to the Kings Esarhaddon and Assurbanipal, State Archives of Assyria 13 (Helsinki: Helsinki University Press, 1998), 12, Text Nr. 8:Vs. 15-Rs. 7.

58. Siehe S. Parpola, Letters from Assyrian and Babylonian Scholars, State Archives of Assyria 10 (Helsinki: Helsinki University Press, 1993), 73, Text Nr. 96:Vs. 1-25 (Brief des Akkullanu an Assurbanipal); siehe außerdem: S. W. Cole, P. Machinist, Letters from Priests to the Kings Esarhaddon and Assurbanipal [wie Anm. 57], 12-14, Texte Nr. 8-11 und 20-22, Texte Nr. 18-21.

59. S. W. Cole, P. Machinist, ebd., 20, Text Nr. 18:Vs. 1-15.

60. Vgl. z. B. S. W. Cole, P. Machinist, ebd., 127, Text Nr. 156.

61. Siehe S. Reynolds, The Babylonian Correspondence of Esarhaddon and Letters to Assurbanipal and Sîn-šarru-iškun from Northern and Central Babylonia, State Archives of Assyria 18 (Helsinki: Helsinki University Press, 2003), 109, Text Nr. 133, Rs. 2'-3'. 